\section{Renaissance Quarterly}

The Renaissance Society of America

The Graduate School and University Center

The City University of New York

365 Fifth Avenue, Room 5400

New York, NY 10016-4309

Address Service Requested
Periodical Postage Paid at NEW YORK, NY and additional mailing offices

USPSA FSM 100 Approved Poly. 